\title{
Chain configurations in light nuclei
}

\author{
S. Yu. Torilov* and K. A. Gridnev \\ Department of nuclear physics Saint-Petersburg State University, Saint-Petersburg, Russia
}

\begin{abstract}
The model of nuclear matter built from $\alpha$-particles is proposed. The strong deformed shape for doubly even $\mathrm{N}=\mathrm{Z}$ nuclides from ${ }^{12} \mathrm{C}$ to ${ }^{24} \mathrm{Mg}$ has been determined according to this model.

PACS numbers: 21.60.Ev, 21.60.Gx.
\end{abstract}

\section{INTRODUCTION}

In spite of considerable efforts to find nuclear chain configurations, i.e. nuclei consisting of $\alpha$ particle chains, today it is still not clear if such states exist, except for the nucleus ${ }^{8}$ Be. Since the end of the sixties this issue has been widely investigated, both theoretically and experimentally [1]. Most of the predictions and experimental results were related, as a rule, to highly excited states of light nuclei ${ }^{12} \mathrm{C},{ }^{16} \mathrm{O},{ }^{20} \mathrm{Ne}$ and ${ }^{24} \mathrm{Mg}$, though there were attempts to consider heavier nuclei [2]. Nevertheless, complications of many-particle problems, which arise due to inapplicability of the shell model, as well as unverified interactions in such systems made theoretical predictions insufficiently accurate. On the other hand, high density of those light nuclei, which decay predominantly through $\alpha$-channel, made it hard to compare predictions and the experimental data. In most cases chain configurations are searched at the levels lying above the $\alpha$-fragmentation threshold simply because cluster levels imply energies that exceed the separation energy of one cluster. In this case the role of the lower chain states is played by Ikeda diagram [3], which presents fragmentation energies of light nuclei for all possible $\alpha$-cluster states. The most interesting in this respect is the level $0_{2}^{+}$in the ${ }^{12} \mathrm{C}$ nucleus, which almost exactly matches the energy of decay into three $\alpha$-particles and decays into ${ }^{8} \mathrm{Be}$ and one $\alpha$-particle. This allows a treating of this level as the first level in the rotational band formed by the chain configuration.

\section{MODEL OF BINDING $\alpha$-PARTICLES}

In this paper we undertake another approach, which assumes the existence of low lying chain configurations. At the heart of this approach lies the model of binding $\alpha$-particles [4, 5]. The nucleus in this model consists of interacting $\alpha$-particles, and the sum of interaction energies gives the binding energy of the nucleus. The coordinates of $\alpha$-particles in the nucleus are kept frozen, and their positions are calculated under the following requirements:

1) The binding energy must be at the minimum,

2) The nucleus must have a nearly spherical form.

The $\alpha$-particle formation proceeds due to the fluctuations. Inside of the nucleus, the proton and neutron pairs are correlated dynamically by the proton-neutron interaction Ref. [4, 6]. In Fig. 1 is shown the distribution of the distances between that pairs for the case then this interaction is very small (i.e. for random distribution of the protons and neutrons in the nuclear volume). One can see that for light nuclei we have approximately "equidistant" distribution with RMS $\sim 2 \mathrm{fm}$. In analogy with the Bernal's model of liquid [7] we assume that at low excitation energies the structure

*Electronic address: torilov@nuclpc1.phys.spbu.ru 
TABLE I: Excitation energy in MeV for deformation with $\omega \mathrm{N}_{\alpha}: \mathrm{N}_{\alpha}: 1$. MBA- model of binding $\alpha$-particles, LD- liquid drop model Ref. [11], Chain - Energy of the $\mathrm{N}_{\alpha}$ fragmentation.

\begin{tabular}{lllll}
\hline \hline $\mathrm{N}_{\alpha}$ & Nucleus & MBA & LD & Chain \\
\hline 3 & ${ }^{12} \mathrm{C}$ & 3.0 & 1.0 & 7.27 \\
4 & ${ }^{16} \mathrm{O}$ & 8.98 & 9.0 & 14.44 \\
5 & ${ }^{20} \mathrm{Ne}$ & 12.99 & 13.0 & 19.17 \\
6 & ${ }^{24} \mathrm{Mg}$ & 21.87 & 20.0 & 28.48 \\
\hline \hline
\end{tabular}

of "dynamical" $\alpha$-particles does not change. In this way we come to the quasi-crystal model of the nucleus, where $\alpha$-particles form a lattice [8]. As shown by Hafstad and Teller, the zero-point energy per bond is quite constant for one quasi-crystal structure to another [5], and this also holds for four particles in a row [9]. In Ref. [10] it was shown that the interaction energy of $\alpha$-particles can be described by a wide class of potentials, adequate for atom-atom type of interactions. We found that the appropriate interaction potential is in the form of the simplest one-parameter Yukawa form:

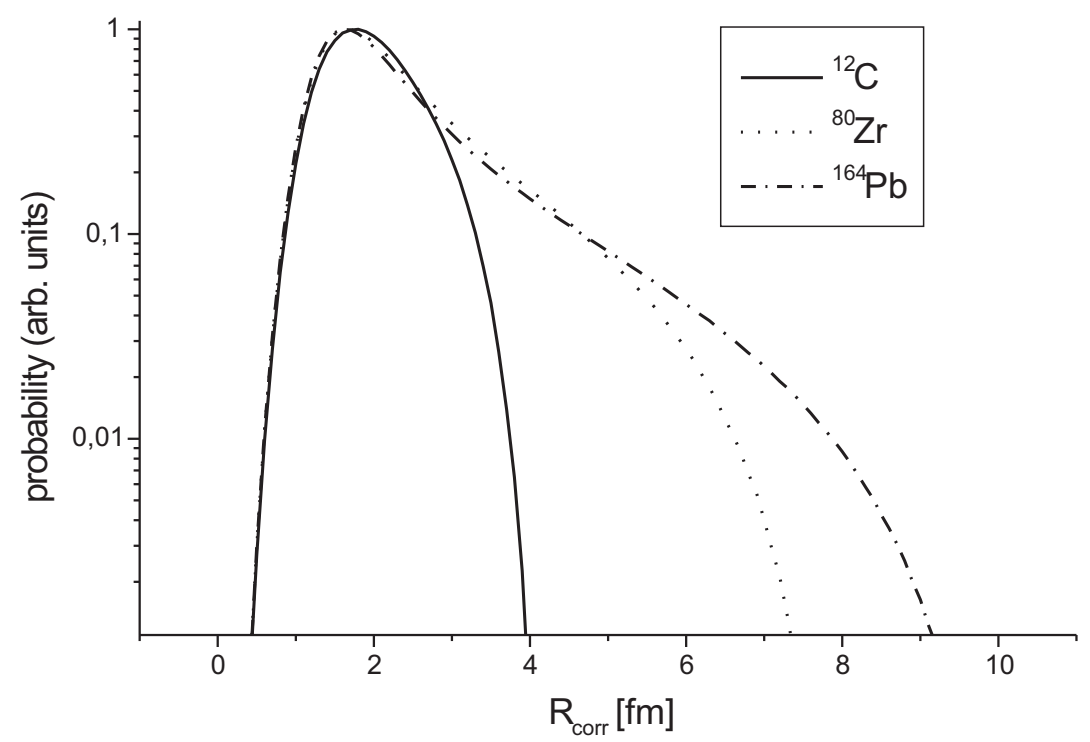

FIG. 1: The distribution of the distances between proton and neutron pairs which form an alpha molecules at a moment. The approach of the random positions the neutrons and protons where used.

$$
V_{\text {nucl }}(r)=-V_{0} * \exp (-\gamma r) / r,
$$

where $\mathrm{V}_{0}$ is fitted through the experimental data [10], $\gamma$ is inverse value of the Compton wavelength of the neutral $\pi$-meson.

In Ref. [10] was shown that fragmentation energies from this interaction potential are in a good agreement with experiment for the light nuclei and qualitatively reflect the behavior of binding 
energies up to the stability border. We are tempting to apply this model to description of chain configurations, being inspired by its success in quantum mechanical description of other nuclear exotics, namely the Bose-Einstein condensation in nuclei [10].

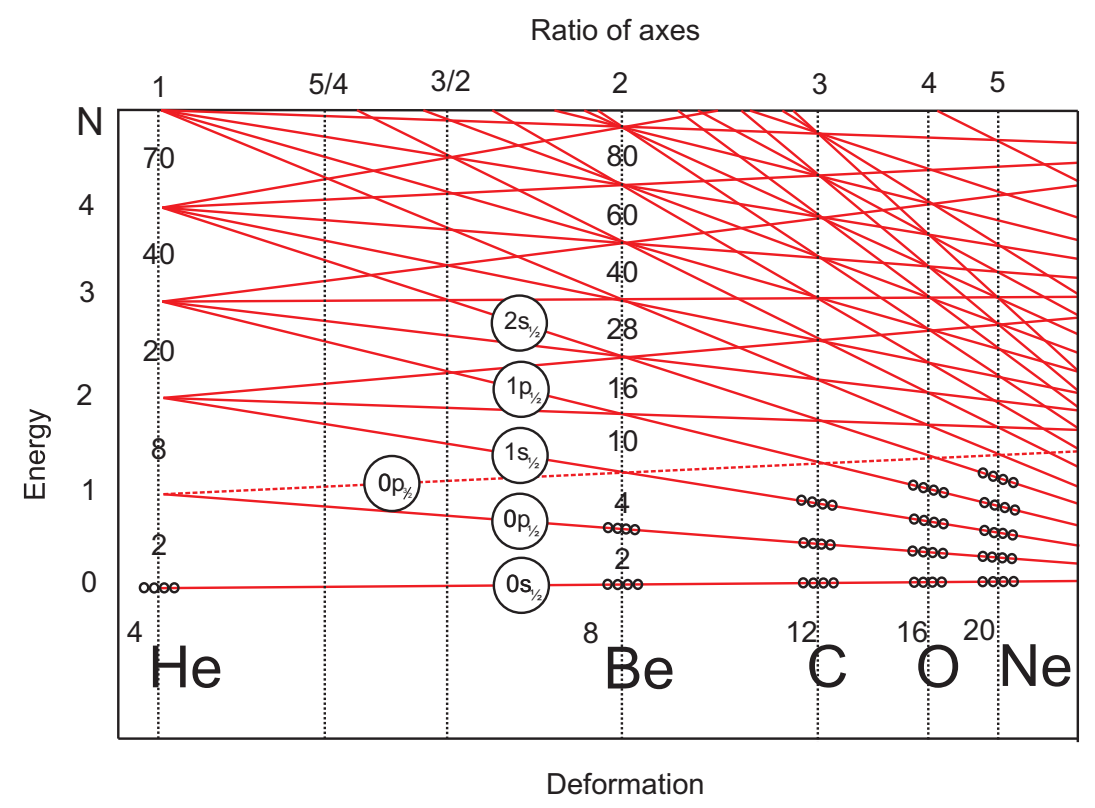

FIG. 2: Shells of the deformed oscillator.

Let us consider the $\alpha$-particle chain, where for interaction we take the sum of Coulomb and nuclear potentials. Then through calculating the binding energy $\mathrm{E}_{b}$ in this system we can find the excitation energy $\mathrm{E}^{*}$, at which the chain may form, from the change of the number of the bonds and the change in the total Coulomb energy [9]. In other words:

$$
E^{*}=E_{f r}-E_{b}
$$

where $\mathrm{E}_{f r}$ is the fragmentation energy. As the parameters of the model we took the depth of the potential $V_{0}$ and the $\alpha$-particle radius $R_{\alpha}$. We should remark that the radius value plays a dual role. On one hand it determines the classical moment of inertia for the $\alpha$-particle. On the other hand it determines the distance between $\alpha$-particles in the chain. We have chosen the value $R_{\alpha}=1.52 \mathrm{fm}$, which is close to the experimental value. The value of $\mathrm{V}_{0}(106.7 \mathrm{MeV})$ was fitted through fragmentation energies [10]. Thus, the energy $\mathrm{E}^{*}$ and the chain's moment of inertia determine the corresponding rotational band. In Tab. I the numerical results for $\mathrm{E}^{*}$ from this study are compared with results from work [11] which have been determined by the macroscopic-microscopic model. It is noteworthy that a shape, corresponding to a linear alignment of $\alpha$-particles, is strongly favored in all double even $\mathrm{Z}=\mathrm{N}$ nuclei. In the harmonic-oscillator description this comes about because the lowest rising levels crosses with the lowest falling level from the $\mathrm{N}^{\text {th }}$ shell when the axis ratio is $\mathrm{N}_{\alpha}: 1$ (see Fig. 2). Therefore we can expect some accordance for these two models. Because the chain rotates around its center of mass and consists of spinless particles it is invariant with respect to reflection and hence the band would have positive parity with even values of the angular momentum. All other levels are distributed according to the standard rule:

$$
E_{J}=\frac{J(J+1) \hbar^{2}}{2 \theta}+E^{*}
$$


where $\mathrm{J}$ is angular momentum and $\theta$ is the moment of inertia. We would like to stress one important point. From the analysis of the $\alpha$-particles rotational band it follows that the moment of inertia is not constant and may increase due to the centrifugal force or decrease due to the reduction in the node number of the wave function [12]. From our simple model we cannot derive the maximum allowed angular momentum or any restriction on the fluctuations of the moment of inertia. As a first approximation we assume that the moment of inertia remains unaltered up to the fragmentation energy. As it was demonstrated in Ref. [13], for the high excitation energy the vibrational degrees of freedom are important, it gives an analogy with molecular spectra.

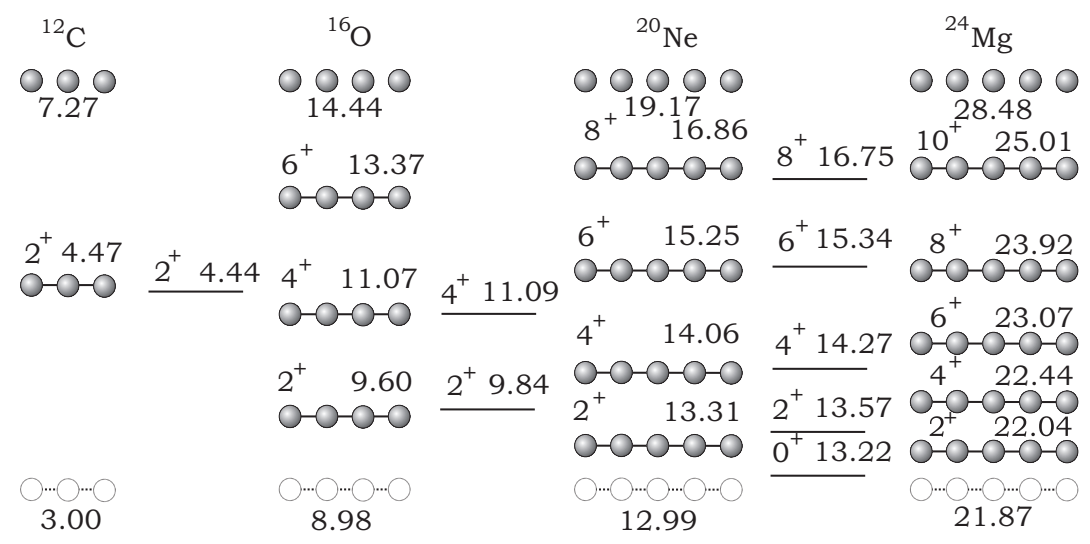

FIG. 3: Theoretical (spheres) and experimental (solid line) energies of rotational bands of chain configurations in nuclei ${ }^{12} \mathrm{C}$, ${ }^{16} \mathrm{O},{ }^{20} \mathrm{Ne}$ and ${ }^{24} \mathrm{Mg}$ (the theory only). Below are the values of zeros for the first levels of rotational bands, upperline corresponds to the fragmentation energy.

Now, to calculate the levels of the rotational band we should know the moment of inertia of the chain configuration. This is calculated according to classical mechanics:

$$
\theta_{N}=\sum_{i=1}^{N} M_{\alpha} a_{i}^{2}+\frac{2}{5} N M_{\alpha} R_{\alpha}^{2},
$$

where $\mathrm{N}$ is the number of $\alpha$-particles, $\mathrm{M}_{\alpha}$ is the $\alpha$-particle mass and $\mathrm{a}_{i}$ are the distances from $\alpha$-particles to the center of mass of the chain configuration.

Fig. 3 shows the obtained energy values for rotational bands in the interval $\mathrm{E}^{*} \leq \mathrm{E}_{J} \leq \mathrm{E}_{f r}$. One finds a good agreement with the experimental values, which have the spin values "required" by the model. The first point to note is that there are no experimental candidates for the predicted $0^{+}$ levels in ${ }^{12} \mathrm{C}$ and ${ }^{16} \mathrm{O}$. As it is well known that the $0_{2}^{+}$state in ${ }^{12} \mathrm{C}$ (Hoyle state) has an excitation energy of $7.65 \mathrm{MeV}$ and there is no evidence of low laying $0^{+}$level at $3 \mathrm{MeV}$. Anywhere calculation predict for this nucleus an oblate ground state and prolate excited state [11] in agreement with our results. In ${ }^{16} \mathrm{O}$ the predicted level possible has the same energy as $2^{-}(8.87 \mathrm{MeV})$ state. A substantial deviation happens for the level $6^{+}$in ${ }^{16} \mathrm{O}$. This could be explained as follows. As noted above, in this model we cannot give an exact quantum mechanical value of the maximum momentum as it is usually done with the help of the Wildermuth condition. Yet, one can determine the maximum angular momentum from classical considerations. The angular frequency of the rotating deformed nucleus is

$$
\omega=\frac{\hbar(J(J+1))^{1 / 2}}{\theta}
$$




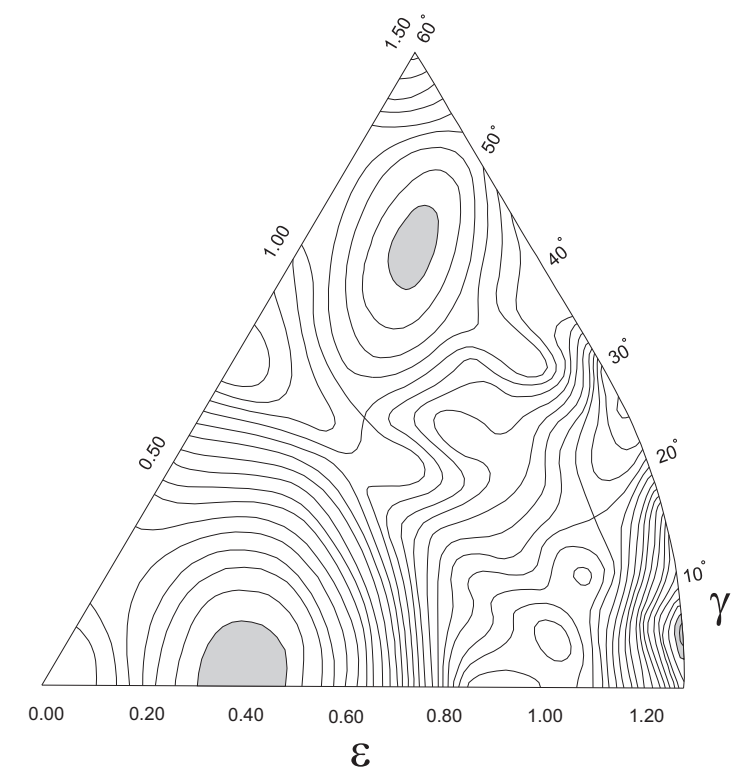

FIG. 4: The potential-energy surface for ${ }^{20}$ Ne Ref. [11].

When one calculates the values of $\omega$ for considered nuclei, one finds that the frequencies corresponding to maximal angular moments are nearly equal to 0.8-1.0 MeV. Thus the adiabaticity condition in this model $\omega_{\text {rot }} \ll \omega_{\text {osc }}$ is fulfilled to a reasonably good extent and a higher angular momentum would break it. As was shown in [15] the maximal angular momentum of the hyperdeformed nucleus ${ }^{152}$ Dy is around $100 \hbar$. According to [16] this value is in good agreement with drop model's prediction for rotation nuclei. The radius of nuclei is proportional to $\mathrm{A}^{1 / 3}$, so the same velocity on the surface leads to maximal angular momentum for nuclei ${ }^{12} \mathrm{C},{ }^{16} \mathrm{O}$ and ${ }^{20} \mathrm{Ne}-3,5$ and 7 which is in agreement with the experiment. Unfortunately, it is impossible to calculate the forces within this model directly because we use the model of a hard rotator, which is obviously invalid for higher angular momenta. Nevertheless, the forces resulting from the above potential are close by the order of magnitude to proclaimed values. In principle this model is capable of reproducing not only rotational levels of the chain configurations but all cluster levels in the Ikeda diagram, for example, for the rotational band of ${ }^{16} \mathrm{O}$. The excitation energy of two protons and two neutrons from the $p$-shell to the $s d$-shell gives rise to a very large triaxial deformation. As was shown in [18] this oblate shape is similar to the $\mathrm{C}_{4}$-symmetric shape (four $\alpha$-particles are placed in a plane forming a square). For this shape the calculation gives the excitation energy of around $6 \mathrm{MeV}$ and the parameter $\hbar^{2} / 2 \theta$ of around $0.22 \mathrm{MeV}$, so we have a good agreement with experimental data. A too low excitation energy of chain configurations alerts, when one compares it with fragmentation energy. However, one should keep in mind that sometimes the $\alpha$-particle bands in light and intermediate nuclei start deeply below the threshold of one $\alpha$-particle separation, i.e. below the corresponding value on the Ikeda diagram. The most interesting from this point of view is the nucleus of ${ }^{20} \mathrm{Ne}$. In Tab. I there are the parameters of the levels [17], which are possible form the rotational band. Like for ${ }^{24} \mathrm{Mg}$ rotational band in [14] we can consider the potential-energy surface for ${ }^{20} \mathrm{Ne}$. We can see [11] that really there are some minima in this potential surface and one of these have strong prolate deformation (see Fig. 4). For this nucleus every $0^{+}$state correspond to the rotational band (see table 20.20 in [17]). Therefore, as is shown in Fig. 5 we have 7 rotational bands and some of them are described in terms of liquid drop model and correspond to the locals minima on Fig. 4. The rotational band which was discussed above corresponds to $0_{8}^{+}$and has $\mathrm{J}(\mathrm{J}+1)$ dependence. In conclusion, we would like to note that these predictions are need in the experimental confirmation. 
TABLE II: Rotational band $\mathrm{K}=0_{8}^{+}$in ${ }^{20} \mathrm{Ne}$.

\begin{tabular}{lll}
\hline \hline $\mathrm{J}$ & $\mathrm{E}^{*}[\mathrm{MeV}]$ & $\Gamma[\mathrm{keV}]$ \\
\hline 0 & 13.22 & 40 \\
2 & $13.53 / 13.57$ & $61 / 12$ \\
4 & 14.27 & 92 \\
6 & 15.35 & - \\
8 & 16.75 & 160 \\
\hline \hline
\end{tabular}

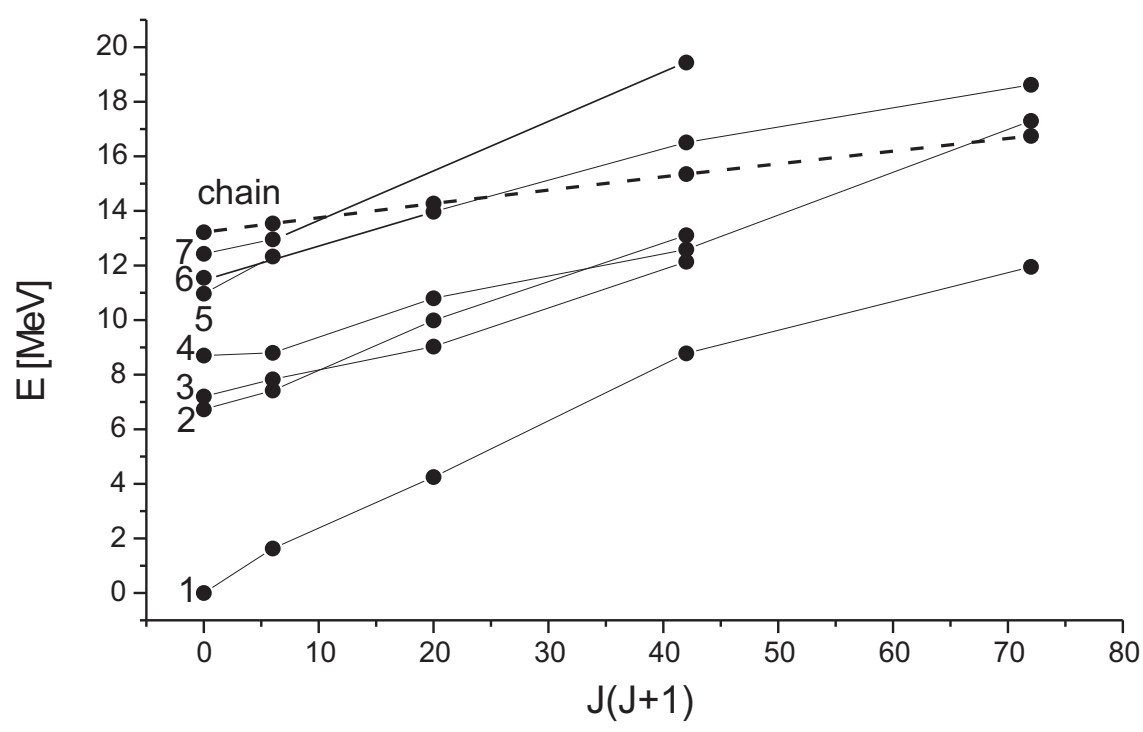

FIG. 5: The rotational bands for ${ }^{20} \mathrm{Ne}$ Ref. [17].

\section{Acknowledgments}

The work was done under the support of the Russian Federation President's Grant MK-1897.2005.2

[1] D. M. Brink, E. Boeker, Nucl. Phys. A91, (1967), 1

[2] A. C. Merchant, W. D. M. Rae, Nucl. Phys. A549 (1992) 431; J. Phys. G19, (1993) L89

[3] H. Horiuchi, K. Ikeda, Y. Suzuki, Suppl. Progr. Theor. Phys. 52 (1972) 89

[4] J. A. Wheeler, Phys. Rev. 52 (1937) 1083

[5] L. R. Hafstad, E. Teller, Phys. Rev. 54 (1938) 681

[6] S. Koh Progr. Theor. Phys. Supl. 132(1998) 197

[7] J. D. Bernal, Nature 183 (1960) 141; Ibid 185 (1960) 68

[8] K. A. Gridnev, S. Yu. Torilov, et. al. Int. J. of Mod. Phys. E 14 (2005) 635

[9] H. Morinaga, Phys. Rev. 101 (1956) 254

[10] K.A. Gridnev, S.Yu. Torilov Phys. Atomic Nuclei 69 (2006) 1204

[11] G. Leander, S.E. Larsson Nucl.Phys. A239 (1975) 93

[12] B. Buck, C. B. Dover, J. P. Vary, Phys Rev C11 (1975) 1803

[13] D. M. Dennison, Phys. Rev. 57 (1940) 454

[14] M. Freer, A. C. Merchant J. Phys. G 23 (1997) 261

[15] A. Galindo-Uribarri, et al., Phys. Rev. Lett. 71 (1993) 231

[16] A. Boucenna, et al., arXiv nucl-th/0305026

[17] D. R. Tilley, et al., Nucl.Phys. A636 (1998) 249

[18] S. Abeg and L-O Jonsson, Z.Phys. A 349 (1994) 205 